\title{
Localization of Carpophore Initiation in Coprinus congregatus
}

\author{
By IAN K. ROSS \\ Department of Biological Sciences, University of California, Santa Barbara, California 93106. \\ U.S.A.
}

(Received 28 October 1981: revised 21 April 1982)

\begin{abstract}
The differentiation of mushroom primordia in young mycelia of Coprinus congregatus is a localized event : the mycelium is not uniformly competent to differentiate, or even to be induced, in all areas. A narrow zone corresponding to the $24 \mathrm{~h}$ growth of the youngest hyphae is the only area capable of receiving the obligatory light induction and of eventually differentiating into primordia. The inducible zone migrates outwards with the peripheral hyphae as the mycelium expands and previously competent zones become inactive and non-inducible. Light induction of a zone fixes the zone of differentiation in space and prevents subsequent zones from becoming induced. The light receptor system appears to involve two independent processes. One is activated by very low levels of light, fixes the zone in space and prevents subsequent zones from becoming induced, but does not permit differentiation. A second, requiring higher light levels, triggers development of the primordia. The induction of a responsive zone can occur at $20^{\circ} \mathrm{C}$, but actual development requires $25^{\circ} \mathrm{C}$.
\end{abstract}

\section{INTRODUCTION}

Coprinus congregatus Bull. ex Fr. is a small mushroom-forming basidiomycete that can complete its life cycle from spore to spore in $12 \mathrm{~d}$ on agar media (Ross, 1979). It is heterothallic and mating is controlled by a unifactorial, multiple allele incompatibility system (Raper, 1966) with at least six alleles known (Ross, 1979). The formation of basidiocarps (mushrooms) is influenced by light (Manachère, 1970) and probably by nutrient levels (Moore et al., 1979).

Many environmental factors can affect or regulate differentiation in the basidiomycetes (see Taber, 1966; Hayes, 1978; Tan, 1978), but the actual mechanisms remain unknown. One possible reason may be that most studies have employed whole mycelia, but different parts of mycelia grown on solid substrates can vary tremendously in their physiological responses, the 'fairy ring' formation of mushrooms in the field being but one example of this. Such localization means that whole mycelia studies are of little value in determining the nature of receptor and regulatory systems. Rusmin \& Leonard (1975) showed that a diffusible fruiting-inducing substance had an effect only on a narrowly delimited zone of new hyphal growth and not on older parts of the mycelium of Schizophyllum commune. It is important, therefore, to use organisms in which the localization of differentiation can be determined accurately and to define conditions that lead to predictable areas of differentiation. In this paper, I report a localization of carpophore initiation in $C$. congregatus that is affected by culture age, levels of nutrients, temperature and light.

\section{METHODS}

Organism and culture. Coprinus congregatus was isolated from commercial steer manure in 1971 and has been maintained as homokaryons derived from single spores on Difco Emerson's YpSs medium (Ross, 1979). The particular homokaryons used in this study have been stored as mycelia at $4{ }^{\circ} \mathrm{C}$ and transferred once a year. Cultures were established from the stocks 1 month before experimentation began and the two homokaryons crossed to form the dikaryon 2 weeks before the first series of experiments. The two homokaryons used were cc8 
(mat 2, pal ${ }^{-}$) and ccl6 (mat 1, pal $^{-}$). The mat locus is the mating type locus and the pal ${ }^{-}$gene is recessive for a meiotic inhibition (Ross, 1982a). The homozygous recessive pal genotype of the dikaryon (cc8 $\times$ cc16) was chosen to prevent the formation, release and germination of basidiospores from affecting cultures undergoing long-term studies. All cultures were grown on $30 \mathrm{ml}$ agar in $100 \mathrm{~mm}$ diameter Petri dishes at $25^{\circ} \mathrm{C}$ in a regime of $12 \mathrm{~h}$ light/12 h dark (L/D), unless otherwise noted. Light was supplied by a bank of five cool-white fluorescent lamps at a distance of $90 \mathrm{~cm}$. All inoculations were performed by removing a plug of agar from the centre of fresh agar plates with a $5 \mathrm{~mm}$ diameter cork borer and replacing it with a similar plug cut from the dikaryon stock. This eliminated exposed cut edges of the inoculum, which can be a preferred site for primordium formation (Leslie \& Leonard, 1979).

Experimental treatment. All light-grown cultures were placed in large plastic boxes (about 150 plates each) in temperature-controlled incubators. All dark-grown cultures were placed in opaque boxes that had been tested for light tightness by exposing Kodak Tri-X film inside for $3 \mathrm{~d}$. 'Full light' was $12 \mathrm{~h}$ of exposure to the fluorescent lamp. 'Low light levels' were achieved by wrapping plastic boxes in a single layer of heavy 'Kraft' brown wrapping paper. Growth rates, and the timing of induction and development were the same for cultures grown under $L / D$ and under constant dark (CD).

All manipulations of dark-grown cultures were done in a darkroom illuminated by a single dissecting microscope lamp covered with a Kodak Wratten No. 58 (green) filter. Exposure to this light does not induce any differentiation in this strain of $C$. congregatus.

For purposes of delimiting growth and induction areas, each day's growth of hyphae (the expanding periphery of the mycelium) was termed a zone, and labelled zone I, zone II, etc. Zone VII was the last zone to be formed and represents the final day's growth prior to reaching the edge of the Petri dish. All experiments were performed at least four times and all treatments were done on 5-10 replicate Petri dishes.

\section{RESULTS}

\section{General growth and development}

Newly inoculated dikaryons grow about $3 \mathrm{~mm}$ in the first $24 \mathrm{~h}, 4 \mathrm{~mm}$ the second $24 \mathrm{~h}$ and then uniformly at a rate of 6-7 mm every $24 \mathrm{~h}$. Peripheral hyphae reach the edge of the Petri dish (edge contact) towards the end of the seventh day of growth.

The overall development of the basidiocarps produced by cultures grown under L/D conditions is shown in Fig. 1. L/D-grown cultures always form primordia (Fig. $1 a$ ) in a zone that represents the hyphae that grew during the third day. Hyphae of the first two days' growth are apparently not inducible. The cultures were examined every $24 \mathrm{~h}$ and primordia would first be seen as minute tufts of hyphae (about $0 \cdot 1 \mathrm{~mm}$ ), similar to those at the left of Fig. $1(a)$.

\section{Differentiation and the timing of the light exposure}

Cultures grown in the dark and exposed to a single $12 \mathrm{~h}$ light period form primordia in a definite relationship to edge contact, as shown in Fig. 2. Regardless of when an inducing light period was given prior to edge contact, no primordia formed until after edge contact and then all cultures induced before edge contact formed primordia at the same time, approximately $24 \mathrm{~h}$ after edge contact. If light induction was given after edge contact primordia formed $24 \mathrm{~h}$ after the beginning of the light period. In $100 \mathrm{~mm}$ diameter Petri dishes containing $30 \mathrm{ml}$ medium, primordia did not form until after both edge contact and a light induction period.

\section{Spatial localization}

Of particular significance to the question of localization was the actual position of the primordia induced in the experiments shown in Fig. 2. Figure 3 shows cultures exposed to light at $24 \mathrm{~h}$ intervals from the end of day 3 onwards. The zone of primordium differentiation was approximately the same as the $24 \mathrm{~h}$ growth zone prior to the exposure. As the cultures grew in the dark, the responsive zone expanded with the periphery of the mycelium. Through to day 7 , therefore, it was the youngest hyphae that were capable of responding. Primordium formation on days 8-10 did not seem to follow a particular pattern and discrete zonation disappeared.

The cultures shown in Fig. 3 were grown on $30 \mathrm{ml}$ medium per dish. If the volume of medium was reduced to $15 \mathrm{ml}$ per dish, the zonation pattern changed dramatically, as shown in Fig. 4 . Here, regardless of when the inducing light period was given up to day 6 , only zone III was responsive and formed primordia. There are occasional exceptions, as shown by the thin line of 
(a)

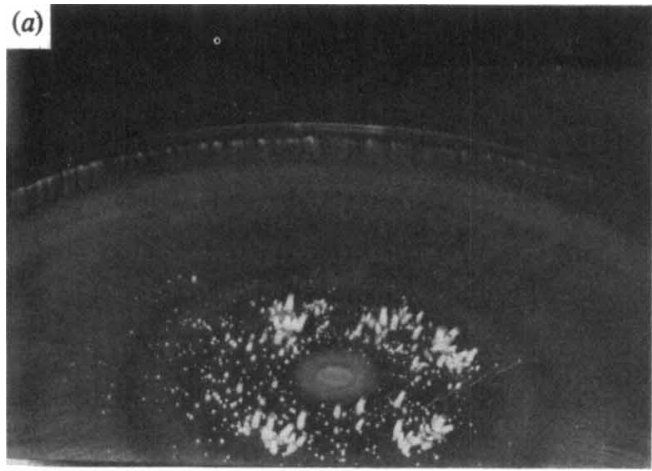

(c)

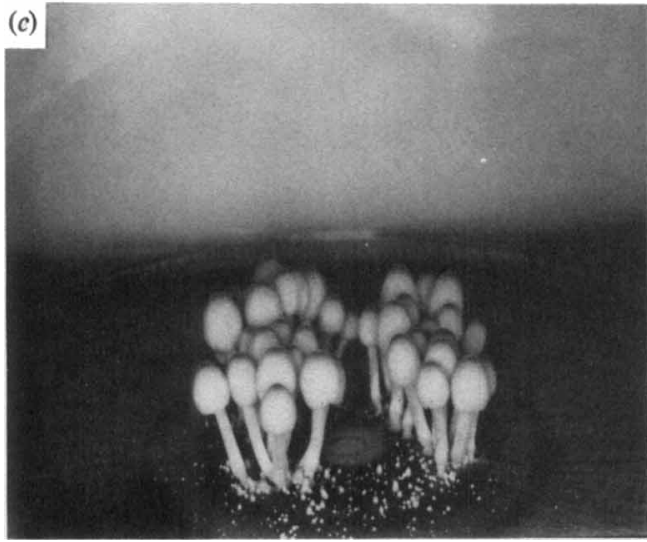

(b)

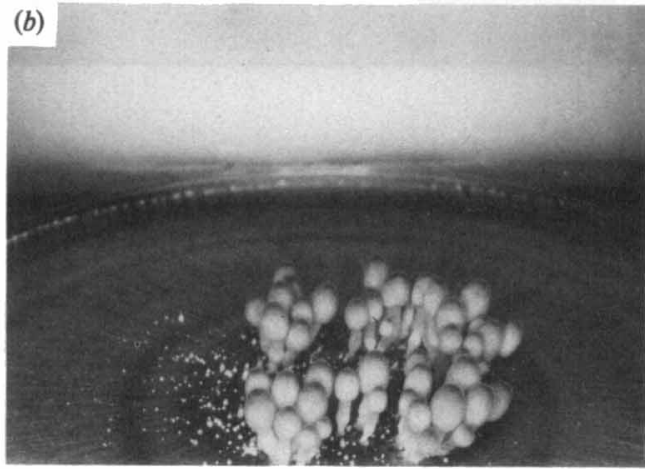

$(d)$

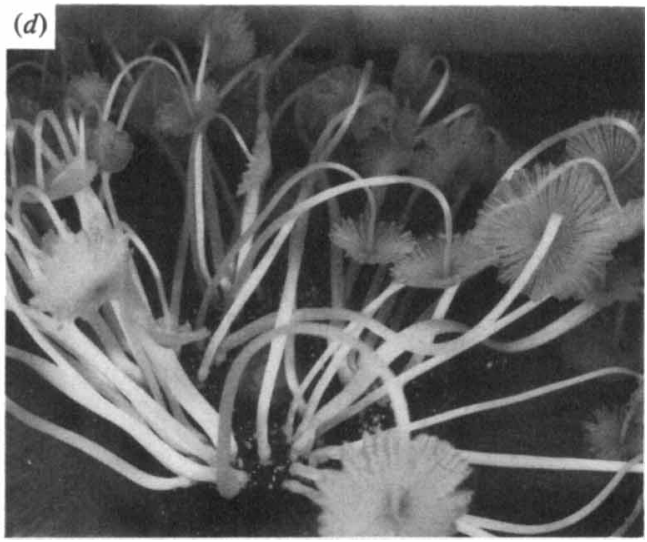

Fig. 1. Coprinus congregatus. basidiocarp development in $150 \mathrm{~mm}$ diameter Petri dishes. (a), $48 \mathrm{~h}$ after edge contact (see text); (b), $24 \mathrm{~h}$ after $(a) ;(c), 12 \mathrm{~h}$ after $(b) ;(d) 12 \mathrm{~h}$ after $(c)$.

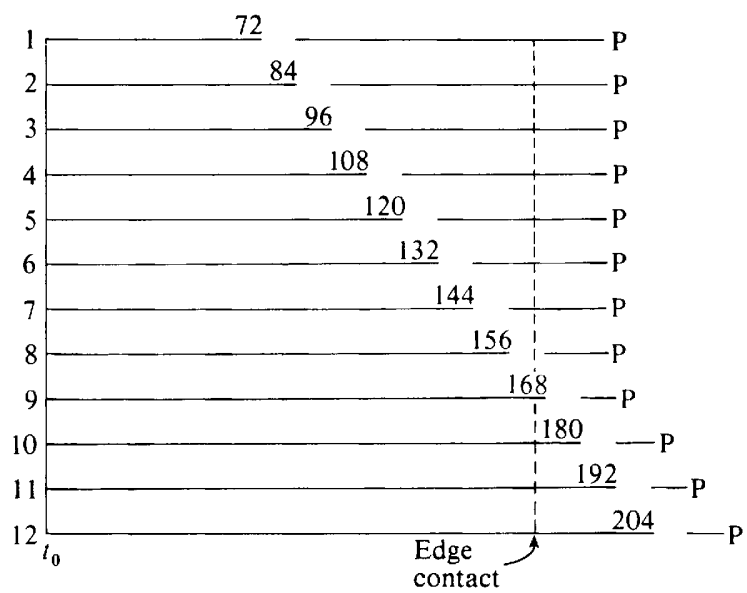

Fig. 2. Culture age and light-induction timing. Twelve sets of cultures (10 plates each) were inoculated at $t_{0}$ (left margin) and grown in the dark as indicated by horizontal unbroken lines. One $12 \mathrm{~h}$ light exposure was given starting at the times (h) indicated at the breaks. $\mathrm{P}$, first sign of primordium appearance.

primordia in zone $\mathrm{V}$ in the culture illuminated on day 4 (Fig. 4 b), but these are rare (less than 1 in 500 plates). Illumination given on day 7 and subsequent days induced a double ring of primordia in zones III and IV only. There was no indication of a gradually expanding zone of inducibility. 

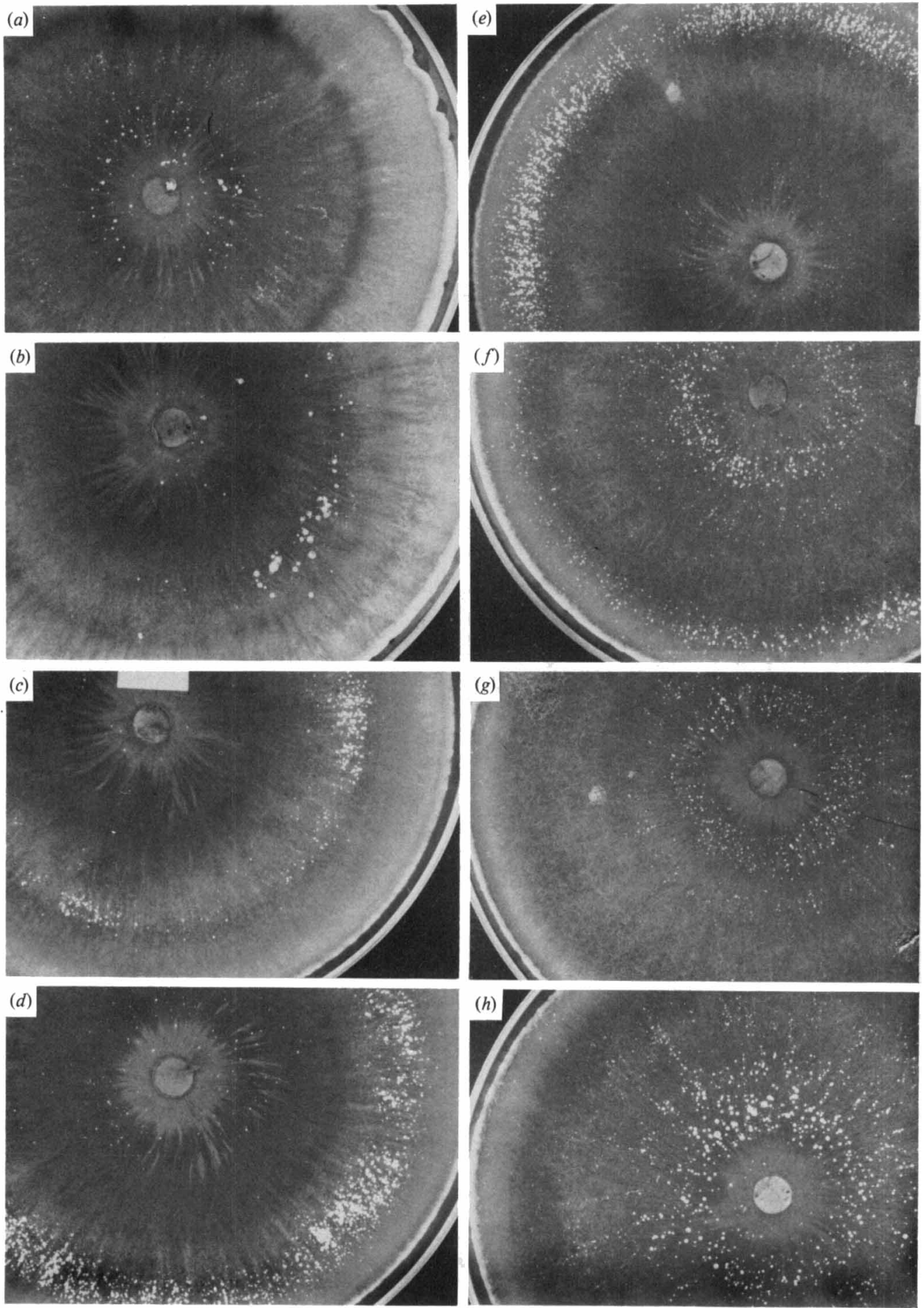

Fig. 3. Localization of primordium formation in plates $(100 \mathrm{~mm}$ diam.) containing $30 \mathrm{ml}$ agar: experiment shown in Fig. 2, exposed to a $12 \mathrm{~h}$ light period after different periods of growth in the dark and photographed after primordia had formed. Cultures were exposed on : (a), day $3(72 \mathrm{~h}) ;(b)$, day $4(96 \mathrm{~h})$; $(c)$, day $5(120 \mathrm{~h}) ;(d)$, day $6(144 \mathrm{~h}) ;(e)$, day $7(168 \mathrm{~h}) ;(f)$, day $8(192 \mathrm{~h}) ;(\mathrm{g})$, day $9(216 \mathrm{~h}) ;(h)$, day 10 (240 h). 

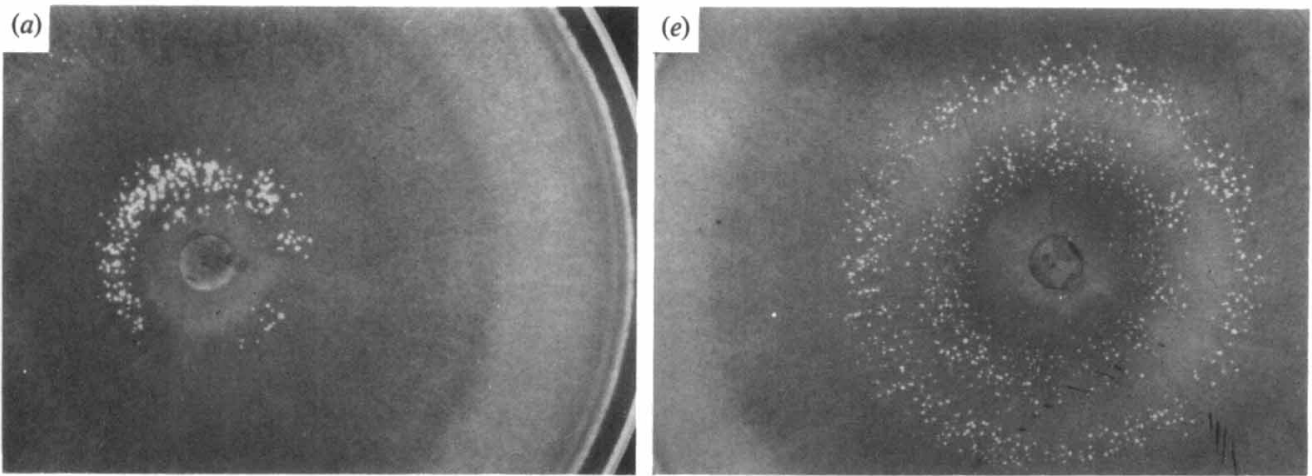

(b)
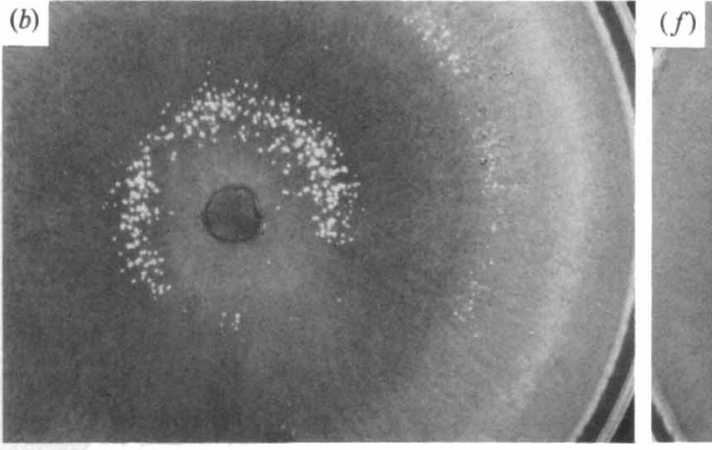

(c)

(g)

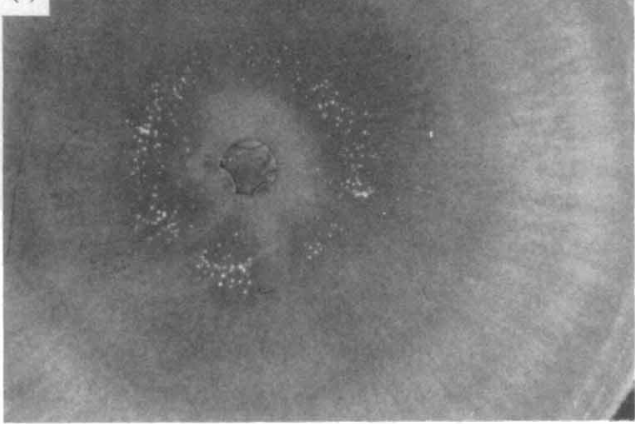

(d)

(h)
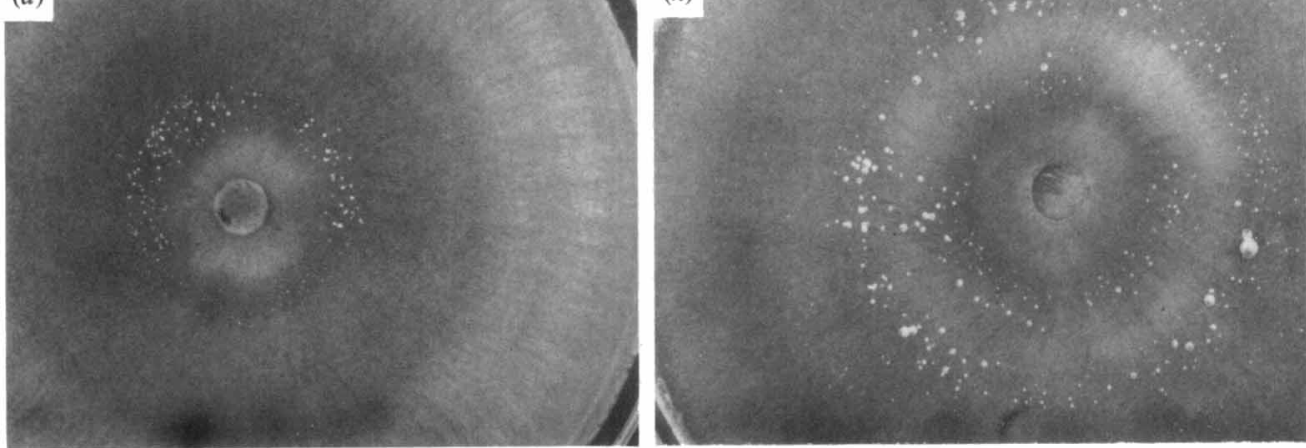

Fig. 4. Localization of primordium formation in plates ( $100 \mathrm{~mm}$ diam.) containing $15 \mathrm{ml}$ agar. The notation for c.lture exposure times is as shown in Fig. 3. 
The experiments shown in Figs 3 and 4 were repeated four times with identical results. The amount of medium appears to be critical for the establishment of discrete, expanding zones.

Returning the cultures induced by a single light period to the dark is not necessary to obtain single zones of primordia. Cultures illuminated as shown in Fig. 2, but placed in L/D conditions after the inducing light period still formed only a single zone of primordia in the appropriate location for the time of the light exposure they had first received.

\section{Temperature effect}

Cultures were inoculated and then incubated in the dark at 20,25 , and $30^{\circ} \mathrm{C}$, in $30 \mathrm{ml}$ agar per plate. They were exposed to a single inducing light period at those temperatures on days 4,5 or 6 , and returned to the dark at the same temperature. The $25^{\circ} \mathrm{C}$ cultures responded as expected and produced zones of primordia in zones IV, V, or VI at the appropriate time ( $24 \mathrm{~h}$ after edge contact). No cultures incubated at $20^{\circ} \mathrm{C}$ or $30^{\circ} \mathrm{C}$ formed primordia. However, if the cultures at $20^{\circ} \mathrm{C}$ were raised to $25^{\circ} \mathrm{C}$ in the dark on day 10 , primordia then formed in the zone that had been illuminated on day 4,5 or 6 . Such single-illuminated cultures grown at $20^{\circ} \mathrm{C}$ could be maintained in the dark at $20^{\circ} \mathrm{C}$ for at least $10 \mathrm{~d}$ after edge contact and still give rise to a zone of primordia corresponding to the zone of illumination when raised to $25^{\circ} \mathrm{C}$ in the dark. The width of the zone of primordia induced at $20^{\circ} \mathrm{C}$ was the same as that induced at $25^{\circ} \mathrm{C}$. Cultures grown at $30^{\circ} \mathrm{C}$ did not produce primordia under any subsequent temperature regimes, no matter when the temperature was lowered after the light period.

\section{Low light effects}

As shown above, dark-grown cultures exposed to $12 \mathrm{~h}$ light and returned to the dark will form a ring of primordia in the growth zone that had been exposed, after the mycelium reaches edge contact. Cultures exposed to $1 \mathrm{~h}$ low-level light did not form primordia in the dark, after the mycelium had reached edge contact (day 8 ). When such short-term or low light induced cultures were re-exposed to $4 \mathrm{~h}$ full light on day 8, primordia appeared in $24 \mathrm{~h}$ on the growth zones present during the low-level exposure.

Dark-grown cultures exposed to $1 \mathrm{~h}$ of low light on day 4 and again on day 6 did not form primordia in the dark by day 8 . When these cultures were re-exposed to $4 \mathrm{~h}$ full light on day 8 , primordia formed in only zone IV. Controls exposed only on day 4, or day 6, or dark grown, formed primordia in zones IV, VI and VII, respectively, when re-exposed on day 8.

\section{DISCUSSION}

The evidence presented confirms the existence of localized zones or areas of inducibility. This reinforces the earlier comment that whole mycelium analysis would be incorrect. Of major interest, as shown in Figs 2, 3, and 4, is that the inducible zone occurs in a predictable manner if certain environmental conditions are known and kept stable. This zone remains essentially the same width and moves out as the mycelium expands. Since primordia can only be induced in such a zone, it is logical to assume that any light receptor system should preferentially be sought there, rather than in other areas of the mycelium.

The relationship of the inducible zone to the growth zone of the previous $24 \mathrm{~h}$, and the constant width of the zone of primordia, whether induced at 20 or $25^{\circ} \mathrm{C}$, suggests that the inducible zone is the same as Trinci's (1971) peripheral growth zone. The peripheral growth zone is the region in which young hyphae are continually entering fresh media and contributing protoplasm to apical extension of the leading hyphae.

If the first zone to become capable of being induced (zone III) is stimulated by light, then no subsequent zone can become induced. This is found to be true of all zones in succession; the most recently formed is the most susceptible, unless a previously formed zone has already been induced. This suggests that the act of light induction, or the reception of light by one zone, effectively prevents any subsequent zone from becoming induced. Conversely, for example, a dark-grown $6 \mathrm{~d}$ old culture, which would have been able to form primordia in zones III, IV, and $\mathrm{V}$ if it had been illuminated on days 3,4 , or 5 , does not do so if illuminated on day 6 . There is the 
strong implication that older zones lose their inducibility as subsequent zones become responsive. Such a loss of inducibility could be caused by decay of the light-receptor system, migration of the system with the growing hyphal perimeter, or active inhibition by the newer zone.

Moore et al. (1979) have discussed the probable relationship between carbohydrate depletion of the medium, increases in glycogen storage in submerged hyphae, and the induction of primordium development in Coprinus cinereus. They showed that a mature, dark-grown, mycelium is in a state of incipient readiness for light induction. Because such studies have used cultures grown long beyond edge contact in the dark, little attention has been paid to the possibility that light may be received and the effect retained prior to nutrient depletion.

As shown in Fig. 2, no primordia formed in the $100 \mathrm{~mm}$ diameter plates until after the mycelium had reached the edge of the plate. Since the hyphae of this fungus extend downwards through the agar to the bottom of the plate only a short way behind the surface peripheral hyphae, it is quite possible that when these hyphae reach the edge of the dish, the nutrients available have been considerably depleted. However, in the absence of definitive studies on the correlation between edge contact and nutrient levels, edge contact is used only as a consistent and predictable way of timing differentiation events.

An induced zone can retain that (induced) state for several days before actual primordium development occurs. This is shown by the differential timing of the light exposure experiment and by the temperature experiment. The act of receiving light in a responsive zone apparently fixes the receptor system into a permanently induced zone that can persist for a considerable period. A similar strict localization of light responses was reported by Galun (1971) in a study of narrow-beam illumination of Trichoderma.

The temperature study also suggests that transduction from light to development is at least a two-step process. The initial step, that of light reception and fixation of the induced zone in place, can occur at low $\left(20^{\circ} \mathrm{C}\right)$ but not at high $\left(30^{\circ} \mathrm{C}\right)$ temperature. A second step, that of the actual initiation of development, does not occur at $20^{\circ} \mathrm{C}$, but can at $25^{\circ} \mathrm{C}$.

A further indication of multiple steps in the light receptor system is indicated by the low-level exposure experiments. Here temperature was not a factor, since all cultures were grown at $25^{\circ} \mathrm{C}$. A single low-level exposure was insufficient to promote primordium formation, but was sufficient to activate the exposed zone and to prevent subsequent zones from becoming involved.

The scattered, almost random, appearance of primordia on older plates illuminated after edge contact suggests that the strict localization of the receptor system no longer exists. Once the mycelium reaches the edge of the Petri dish, there are no longer any zones of actively growing young hyphae. It is probable that once this state has been reached, random hyphal tips in older parts of the mycelium may be induced by local micro-environmental changes to start growing, and by so growing to become 'young' and thus responsive.

The pattern of zonation found in cultures containing only $15 \mathrm{ml}$ of medium in contrast to those containing $30 \mathrm{ml}$ will be discussed in the accompanying paper (Ross, 1982 b).

The support of the Faculty Research Committee and the assistance of students of the College of Creative Studies at UCSB, M. Elliot, P. House, and K. Murphy, and the expert technical assistance of L. Henderson are gratefully acknowledged.

\section{REFERENCES}

Galun, E. (1971). Morphogenesis in Trichoderma: induction of conidiation by narrow-beam illumination of restricted areas of fungal colony. Plant Cell Physi$\operatorname{olog} y 12,779-783$.

HAYES, W. A. (1978). Cultivated fungi in Agaricales: biological nature. In The Biology and Cultivation of Edible Mushrooms, pp. 191-217. Edited by S. T. Chang \& W. A. Hayes. New York \& London: Academic Press.
Leslie, T. F. \& LeOnaRD, T. J. (1979). Three independent genetic systems that control initiation of a fungal fruiting body. Molecular and General Genetics 171, 257-260.

MANACHÈRE, G. (1970). Recherches physiologiques sur la fructification de Coprinus congregatus Bull. ex Fr.: action de la lumière; rythme de production de carpophores. Annales des sciences naturelles, botanique, 11, $1-96$. 
Moore, D., Elhiti, M. M. Y. \& Butler, R. D. (1979). Morphogenesis of the carpophore of Coprinus cinereus. New Phytologist 83, 695-722.

RAPER, J. R. (1966). Genetics of Sexuality in Higher Fungi. New York: Ronald Press.

Ross, I. K. (1979). An infectious disorder for meiosis in Coprinus congregatus. In Viruses and Plasmids in Fungi, pp. 485-524. Edited by P. A. Lemke. New York: M. Dekker.

Ross, I. K. (1982a). The genetic basis for the meiotic disorder of Coprinus congregatus. Current Genetics $\mathbf{5}$ 53-56.

Ross, I. K. (1982b). The role of laccase in the initiation of differentiation in Coprinus congregatus. Journal of General Microbiology 128, 2763-2770.
Rusmin, S. \& LeONARD, T. J. (1975). Biochemical induction of fruiting bodies in Schizophyllum commune: bioassay and its application. Journal of General Microbiology 90, 217-227.

TABER, W. A. (1966). Morphogenesis in Basidiomycetes. In The Fungi, vol. 2, pp. 387-412. Edited by G. C. Ainsworth \& A. S. Sussman. New York \& London: Academic Press.

TAN, K. K. (1978). Light-induced fungal development. In The Filamentous Fungi, vol. 3, Developmental Mycology, pp. 334-357. Edited by J. E. Smith \& D. R. Berry. New York: J. Wiley.

TRINCI, A. P. J. (1971). Influence of width of the peripheral growth zone on the radial growth rate of fungal colonies on solid media. Journal of General Microbiology 67, 325-344. 